\title{
Immune Privilege of Primary Fetal Progenitor Cells: Optimized Safety Profile of Consistent Cell Sources for Cutaneous and Musculoskeletal Regenerative Medicine
}

\author{
Alexis Laurent ${ }^{1}$, Nathalie Hirt-Burri ${ }^{1}$, Corinne Scaletta ${ }^{1}$, Lee Ann Applegate ${ }^{1,2,3}$ and Anthony $S$ de \\ Buys Roessingh ${ }^{4 *}$ \\ ${ }^{1}$ Musculoskeletal Medicine Department, Lausanne University Hospital, Switzerland
}

${ }^{2}$ Center for Applied Biotechnology and Molecular Medicine (CABMM), University of Zurich, Switzerland

${ }^{3}$ Oxford OSCAR Suzhou Center, Oxford University, PRC

${ }^{4}$ Department of Pediatric Surgery, Lausanne University Hospital, Switzerland

*Corresponding author: Anthony S de Buys Roessingh, Pediatric Surgery Service, Lausanne University Hospital, CHUV, University of Lausanne, CH-1011 Lausanne, Switzerland

To Cite This Article: Alexis Laurent, Nathalie Hirt-Burri, Corinne Scaletta, Lee Ann Applegate, Anthony S de Buys Roessingh. Immune Privilege of Primary Fetal Progenitor Cells: Optimized Safety Profile of Consistent Cell Sources for Cutaneous and Musculoskeletal Regenerative Medicine. 2020 - 8(6). AJBSR.MS.ID.001330. DOI: 10.34297/AJBSR.2020.08.001330.

Received: 眥 May 09, 2020; Published: 渄 May 15, 2020

\begin{abstract}
Numerous heterogenous biological tissues and cell types may be valorized and processed for therapeutic material generation and product development in regenerative medicine applications. Autologous, allogenic or xenogenic cell therapies may generally be considered for transplantation, whereas the immunogenicity of the implanted final products is key in determining treatment parameters. Banked primary fetal progenitor cells constitute highly interesting candidates for allogenic cell therapy approaches, as among numerous other technical advantages, such cell types are devoid of the propensity towards recipient immune response eliciting. Selection of specific gestational ages for fetal organ donation procurement enables the eventual obtention of cultured progeny cell populations consistently retaining specific subsets of MHC antigens, allowing for evasion or modulation of recipient immune systems, as attested by transplantation of fetal progenitor cells in allogenic and xenogenic frameworks in Switzerland. The present study briefly summarizes the molecular, preclinical and clinical evidence supporting the immune-privileged status of primary fetal progenitor cells and the contribution thereof to the high therapeutic value of such cellular substrates for regenerative medicine applications.
\end{abstract}

Keywords: Cell banking, Cell therapy, Immune privilege, Primary progenitor cells, Regenerative medicine, Tissue engineering, Wound healing

Abbreviations: ATMP: Advanced Therapy Medicinal Product; GMP: Good Manufacturing Practices; HLA: Human Leukocyte Antigen; IDO: Indoleamine 2,3-Dioxygenase; MHC: Major Histo-compatibility Complex; MSC: Mesenchymal Stem Cell; PBB: Progenitor Biological Bandage; TEP: Tissue Engineering Product

\section{Introduction}

Novel approaches of cell therapy and tissue engineering have recently contributed to widespread combined therapeutic development efforts towards optimal restoration and optimization of tissue or organ functions [1-4]. Multifactorial considerations and extensive planning are necessary in view of selecting optimal therapeutic cell sources for product development and clinical translation of cell therapies or tissue engineering solutions. Technological simplicity and robustness in the obtention of the rapeutic biological materials enables the sustainable manufacture of innovative autologous, allogenic or xenogenic regenerative medicine substrates [5-9]. However and most importantly on the clinical side, considerations about immunogenicity of bioprocessed 
and implanted materials should arise very early in the development phases, as the elicited effects after tansplantation may complexify patient management in case of recognition and tentative rejection by recipient immune systems $[6,8]$.

Primary fetal progenitor cells have been demonstrated to optimally adapt to highly robust and extensive biobanking frameworks, enabling establishment of large homogenous multitiered cryopreserved cell banks (Parental, Master and Working Cell Banks) destined for Good Manufacturing Practices (GMP) production of clinical-grade Advanced Therapy Medicinal Products (ATMP) or medical devices [10-15]. Such cell types, which may be traceably isolated under stringent Transplantation Program frameworks following voluntary pregnancy terminations, fulfill numerous technical requirements of industrial-scale biotechnological processing [8-12]. Fetal progenitors have been shown to be stable, consistent, safe and present therapeutic potentials of utmost interest, as they have been observed to stimulate repair or regeneration of various cutaneous or musculoskeletal injuries such as ulcers, deep burns or volumetric soft-tissue losses by putatively exerting paracrine stimulation of wounded tissues [6,7,9,14,16-19]. Absence of tumorigenicity, good cyto or biocompatibility and on-demand availability, rapid proliferation, pre-terminal differentiation and high resistance to oxidative stress are strong advantages of these cell types, yet the paramount clinical advantages conferred by the specific absence of observable immunogenicity allow for pragmatic consideration of such cellular substrates for simple and effective management of acute and degenerative cutaneous and musculoskeletal affections $[12,15]$.

\section{Optimized Organ Donations and High Therapeutic Value of Fetal Progenitors}

A.

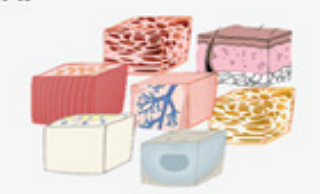

C.

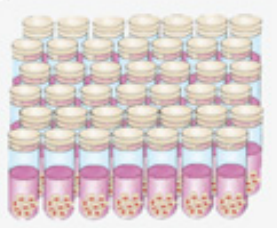

B.

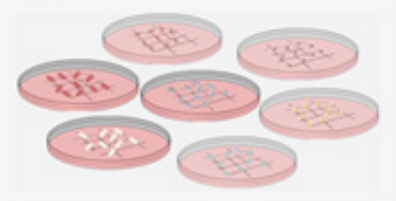

D.

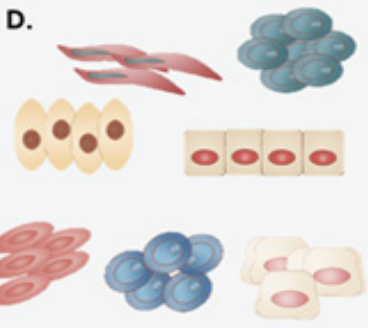

E.

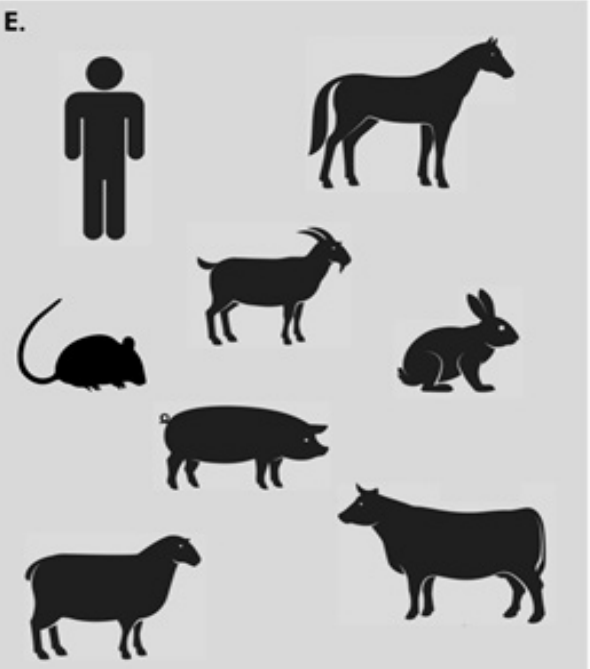

Figure 1: Overview of the establishment of primary fetal progenitor cell types and banking thereof under the Swiss Fetal Progenitor Cell Transplantation Program and types of preclinical or clinical applications documented to date evidencing absence of cell therapy recipient immune system activation. A) Different tissue biopsies are simultaneously provided from one organ donation after micro-dissection. B) Tissues are further dissected into fragments allowing for enzymatic or mechanical cell culture initiation. C) Culture-expanded cell populations are cryopreserved to form homogenous and extensive cell banks. D) Cell vials are thawed and contents may be directly used or further processed in oder to produce cell therapies or cell-based therapeutic products. E) Schematic illustrations of the different patient/animal models which have been treated under the different Swiss Fetal Progenitor Cell Transplantation Programs with primary fetal progenitor cells without presenting signs of immune recognition or rejection to date. All species were treated with human fetal progenitor cells (allogenic treatment, human to human or xenogenic treatment, human to animal) except horses, which were treated with allogenic equine primary progenitor cell types. See Table 1 for specific references.

Table 1: Overview of the different types of cell transplantation (allogenic and xenogenic) using cultured primary fetal progenitor cells after primary cell type establishement and banking in Switzerland. Of all the considered preclinical and clinical applications listed herein, none provided evidence of recipient immune system activation by transplanted therapeutic cellular products.

\begin{tabular}{|c|c|c|}
\hline Type of Transplantation & Example of Applications & References \\
\hline $\begin{array}{c}\text { Allogenic } \\
\text { Human to human }\end{array}$ & Progenitor Biological Bandages for severe burns, donor-site grafts and refractory ulcers & $16-19$ \\
\hline $\begin{array}{c}\text { Xenogenic } \\
\text { Human to animal }\end{array}$ & $\begin{array}{r}\text { Fetal dermal progenitor fibroblasts in porcine models for donor-site grafts } \\
\text { Fetal muscle progenitors in murine models of volumetric muscle loss } \\
\text { Fetal chondroprogenitors in rat subcutaneous models }\end{array}$ & $14,15,20,23,24$ \\
\hline $\begin{array}{c}\text { Allogenic } \\
\text { Animal to animal }\end{array}$ & $\begin{array}{c}\text { Fetal osteoprogenitors in rat models of skeletal volumetric defects } \\
\text { healing }\end{array}$ & 9 \\
\hline
\end{tabular}


Ethical advantages of working with cultured primary progenitor cells are evidenced by the restricted need for fetal biopsy procurement in view of therapeutic product development. It has been established that under the proper bioprocessing frameworks and biobanking methodologies, a single pregnancy termination can allow for the rapid generation of cryopreserved cell stocks potentially serving for subsequent manufacture of billions of therapeutic product units for cutaneous or musculoskeletal regenerative medicine applications $[15,20]$. The significance of such technical capabilities is fully valorized by integrating the clinical aspects of fetal progenitor cell therapy or tissue-engineering product (TEP) applications, as a single universal donor may provide cells for treatment of numerous patients of various immunological dispositions, allowing for maximization of product quality and consistency [15]. The persistant problematic of therapeutic material scarcity or shortages in human organ transplant fields may therefore be avoided in regenerative medicine by the use of widely available non-immunogenic consistent and stable cellular materials such as fetal progenitor cells. Extensive in vitro, preclinical and clinical experience or insights have been gathered around the use of human and animal primary fetal progenitor cells over the last three decades in Switzerland in particular, highlighting the numerous advantages and high therapeutic values of such cell types (Figure 1 and Table 1) [6-25]. Iterative optimization of cell source selection, organ donation procurement and bioprocessing methodologies allow for harnessing of the high therapeutic potential born by primary fetal progenitor cell types $[9,15]$. It has been observed that fetal progenitor cells isolated and cultureexpanded from fetal organ donations ranging between the $12^{\text {th }}$ and $16^{\text {th }}$ week of gestation are characterized by optimal consistency and stability, therefore ensuring maximized safety and product efficacy after adequate bioprocessing and storage $[7,15]$.

\section{Immune Privilege of Fetal Progenitor Cell Types}

Fetal progenitor cell types and mesenchymal stem cells (MSC) display similar specific immunological profiles, as they may be considered to be immune-privileged or classified as possessing immunomodulatory capabilities [13]. It is clear that the gestational age of the fetus at the time of pregnancy termination and organ donation plays a paramount role in the molecular characteristics of derived progeny cultured cells. Indeed, major histo-compatibility complex (MHC) antigen expression during fetal development is organ and gestational age-specific [26]. Fetal progenitor cells are considered pre-immunocompetent and may fail in eliciting immunological responses due to an observed lack of post-thymic $\mathrm{T}$ lymphocytes in the first 13 gestational weeks [5,27]. It is well accepted that $\mathrm{T}$ lymphocytes are heavily implicated in allograft rejection mechanisms, mainly through differential human leucocyte antigen (HLA) recognition, while expression of surface MHC class I proteins typically triggers T CD8-mediated immune reactions [13]. In the particular and comparative case of stem cell studies however, it was shown that MSCs expressing MHC class I antigens could be grafted without eliciting an immunological response. Such cell types have been also shown to have proliferation inhibition capabilities towards T lymphocytes cultured in vitro [28]. Transplantation in primate models has yielded some evidence of low immunogenicity, despite mismatches in MHC panel expression [29,30]. Additionally, neonatal cell types were shown to display similar MHC profiles while presenting acceptable safety (e.g. neonatal foreskin keratinocytes) [31]. By extrapolation, it is probable that primary fetal progenitor cells possess capabilities and behaviors similar to those of MSCs in their interactions with recipient immune system effectors, albeit being different from MSCs in terms of potency [13]. Indeed, primary fetal progenitor cell types generally lack MHC class II proteins (HLADP, DQ DR) and exhibit relatively low levels of MHC class I (HLA-A, B, C) counterparts, therefore closely resembling marker panels yielded by MSCs or neonatal foreskin keratinocytes [13,28,31,32]. Such observed tolerance is highly beneficial for the use of allogenic primary progenitors, as adjuvant immunosuppressive treatments are not necessary after transplantation to immune-competent patients.

Specific fetal tissues were shown to selectively express HLA-G antigens, known mediators of tolerogenic effects [33,34]. Immune-modulation and inhibition of lymphoproliferation by fetal progenitor cells is also attributed to specific expression of indoleamine 2,3-dioxygenase (IDO) [28,30,35]. Fetal cells definitely possess immune system modulation capabilities, as attested during the gestational development, where an immunologic equilibrium exists between the mother and the embryo or fetus. Indeed, absence of immune reaction triggering despite in utero recognition of paternal HLA-C markers and modulatory effects of HLA-G antigens on lymphocytic activity characterize the particular immune status of fetal tissues during gestation and thus that of derived fetal progenitor cells [33,36-38]. Futhermore, it was also shown that fetal cells persist in the mother's tissues for extended periods after delivery and may contribute to healing of maternal injuries [39]. When considering implantation in an immunocompetent recipient, it is possible that multimodal mechanisms are deployed by fetal progenitor cells, leading to localized redefinition of immune microenvironments at the site of delivery, allowing for early inflammation control and stimulation of physiological repair processes [11].

\section{Preclinical and Clinical Immune Tolerance of Fetal Progenitor Cells}

Extensive preclinical experience has been gathered around the use of primary fetal progenitor cells in Switzerland and in subsequent international collaborations [15]. Indeed, cellular materials developed under the Swiss Fetal Progenitor Cell Transplantation Program, comprising various cell types (i.e. fetal dermal progenitor fibroblasts, fetal chondroprogenitors, fetal progenitor tenoyctes, fetal osteoprogenitors or fetal muscle 
progenitors) have been extensively studied [6,8,11,13,14,21 24]. Implantation of human progenitor cells in various xenogenic frameworks (murine, rat, caprine, porcine and rabbit models) have not yielded evidence of immune system activation in immunocompetent animals $[13,15,18,19,23,24]$. Extensive experience around the use of fetal progenitor dermal fibroblasts gathered over two decades for managing burn patients and complicated cutaneous wounds has yieled no evidence of immune recognition of therapeutic allogenic cellular materials, while yielding strong evidence of potent therapeutic stimulation of patient tissues towards restoration of both structure and function $[10,16$ 19]. In particular, Progenitor Biological Bandages (PBB) yielding cultured fetal progenitor fibroblasts have been applied successfully for improving the outcomes of pediatric thermal wounds, autograft donor-sites or geriatric ulcers, promoting tissue regeneration while repeated applications (with absence of allogenic cell engraftment) did not lead to immune system activation $[18,19]$. Moreover, the observed clinical effects after fetal progenitor cell applications rather comprised potent anti-inflammatory components and pain reduction, suggesting an active process or interplay with the recipient organism, rather than a simple passive immune system evasion $[16,19]$. In addition, extended studies and hindsight also indicate an absence of tumorigenicity of primary fetal progenitor cells, which might be due to the inherent relatively high cell stability during processing [15].

\section{Conclusion}

Among the numerous currently available options for cell source selection in therapeutic product research, the immune privilege or immunomodulatory properties of fetal progenitor cells favor their widespread use for tissue engineering or cellular therapy development. Due to inherent characteristics of cultured fetal progenitor cell types, the use of a single donor for the generation of extensive cryopreserved tiered cell banks serving as universal therapeutic material is of great interest for multiple complementary applications in regenerative medicine. The optimal standardization of biological starting materials and the technical simplicity or robustness of fetal progenitor cell types enable effective and sustainable transposition of bioprocessing and manufacturing workflows to GMP production. By selecting specific tissue donor characteristics, defined populations of progenitor cells might be derived without resorting to cell-sorting, while obtained cells express defined subsets of MHC antigens leading them to be devoid of the propensity towards immune response eliciting [15]. Based on the large preclinical and clinical experience around allogenic and xenogenic fetal progenitor cell transplantation in Switzerland, it is possible to safely conclude that the particular immunological status of cultured fetal progenitors constitute a technological strong suit potentially enabling effective, safe and high quality treatment provision to millions of patients suffering from wide arrays of acute and degenerative affections.

\section{Acknowledgements}

We would like to thank the S.A.N.T.E and Sandoz Foundations for their continued commitments to the Swiss Fetal Biobanking Program throughout the years.

\section{Conflicts of Interest}

The Authors have no conflicts of interest to declare.

\section{References}

1. Vacanti JP, Langer R (1999) Tissue engineering: The design and fabrication of living replacement devices for surgical reconstruction and transplantation. Lancet 354 Suppl1: SI32-34.

2. Monti M, Perotti C, Del Fante C, Cervio M, Redi CA, et al. (2012) Stem cells: Sources and therapies. Biol Res 45: 207-214.

3. Marks P, Gottlieb S (2018) Balancing safety and innovation for cell-based regenerative medicine. N Engl J Med 378: 954-959.

4. Loebel C, Burdick JA (2018) Engineering stem and stromal cell therapies for musculoskeletal tissue issue repair. Cell Stem Cell 22: 325-339.

5. Crombleholme TM, Langer JC, Harrison MR, Zanjani ED (1991) Transplantation of fetal cells. Am J Obstet Gynecol 164(1): 218-230.

6. De Buys Roessingh AS, Hohlfeld J, Scaletta C, Hirt-Burri N, Gerber S, et al. (2006) Development, characterization and use of a fetal skin cell bank for tissue engineering in wound healing. Cell Transplant 15(8-9): 823834.

7. Quintin A, Hirt-Burri N, Scaletta C, Schizas C, Pioletti DP, et al. (2007) Consistency and safety of cell banks for research and clinical use: Preliminary analysis of fetal skin banks. Cell Transplant 16(7): 675-684.

8. Applegate LA, Scaletta C, Hirt-Burri N, Raffoul W, Pioletti D (2009) Whole-cell bioprocessing of human fetal cells for tissue engineering of skin. Skin Pharmacol Physiol 22(2): 63-73.

9. Laurent A, Darwiche SE, Hirt-Burri N, Scaletta C, Michetti M, et al. (2020) Banking progenitor cells for hippiatric regenerative medicine: Optimized establishment of safe and consistent cell sources for standardized veterinary therapeutic protocols. 8(4): 252-271.

10. Hirt-Burri N, Scaletta C, Gerber S, Pioletti DP, Applegate LA (2008) Wound-healing gene family expression differences between fetal and foreskin cells used for bioengineered skin substitutes. Artificial Organs 32(7): 509-518.

11. Darwiche SE, Scaletta C, Raffoul W, Pioletti DP, Applegate LA (2012) Epiphyseal chondroprogenitors provide a stable cell source for cartilage cell therapy. Cell Med 4(1): 23-32.

12. De Buys Roessingh AS, Guerid S, Que Y, Berger M, Hirt-Burri N, et al. (2013) Cell therapy assistance in reconstructive surgery for musculoskeletal tissues following burn and trauma: Swiss cellular Transplantation Platform. Def Manag S3: 003.

13. Grognuz A, Scaletta C, Farron A, Raffoul W, Applegate LA (2016) Human fetal progenitor tenocytes for regenerative medicine. Cell Transplant 25(3): 463-479.

14. Laurent A, Hirt-Burri N, Amiot C, Scaletta C, Applegate LA, de Buys Roessingh AS (2020) Primary progenitor muscle cells for regenerative medicine: Standardization of therapeutic protocols and optimized in vivo murine model for volumetric muscle loss. AJBSR 8(2): 143-153.

15. Laurent A, Lin P, Scaletta C, Hirt-Burri N, Michetti M, de Buys Roessingh AS, et al. (2020) Bringing safe and standardized cell therapies to industrialized processing for burns and wounds. Front Bioeng Biotechnol.

16. Hohlfeld J, de Buys Roessingh AS, Hirt-Burri N, Chaubert P, Gerber S, Scaletta C, et al. (2005) Tissue engineered fetal skin constructs for paediatric burns. Lancet 366(9488): 840-2. 
17. Ramelet AA, Hirt-Burri N, Raffoul W, Scaletta C, Pioletti DP, et al. (2009) Chronic wound healing by fetal cell therapy may be explained by differential gene profiling observed in fetal versus old skin cells. Exp Gerontol 44(3): 208-218.

18. Hirt-Burri N, Ramelet AA, Raffoul W, de Buys Roessingh AS, Scaletta C, et al. (2011) Biologicals and fetal cell therapy for wound and scar management. ISRN Dermatol 2011: 549870

19. De Buys Roessingh AS, Hirt-Burri N, Raffoul W, Scaletta C, Applegate LA (2015) A decade after foetal skin progenitor cell therapy in pediatric burn treatment. J Regen Med 4: 1.

20. Hirt-Burri N, de Buys Roessingh AS, Scaletta C, Gerber S, Pioletti DP, Applegate LA, et al. (2008) Human muscular fetal cells: A potential cell source for muscular therapies. Pediatr Surg Int 24(1): 37-47.

21. Montjovent MO, Mathieu L, Hinz B, Applegate LA, Bourban PE, et al. (2005) Biocompatibility of bioresorbable poly (L lactic acid) composite scaffolds with human fetal bone cells. Tissue Engineering 11(11-12): 1640-1649

22. Quintin A, Schizas C, Scaletta C, Jaccoud S, Gerber S, et al. (2009) Isolation and in vitro chondrogenic potential of human foetal spine cells. J Cell Mol Med 13(8B): 2559-2569.

23. Hausherr TC, Nuss K, Thein E, Applegate LA, Pioletti DP (2017) Human bone progenitor cells for clinical application: What kind of immune reaction does fetal xenograft tissue trigger in immunocompetent rats? Cell Transplant 26(5): 879-890.

24. Cavalli E, Fisch P, Formica FA, Gareus R, Linder T, Applegate LA, et al. (2018) A comparative study of cartilage engineered constructs in immunocompromised, humanized and immunocompetent mice. J Immunol Regen Med 2: 36-46.

25. Abdel-Sayed P, Hirt-Burri N, de Buys Roessingh AS, Raffoul W, Applegate LA (2019) Evolution of biological bandages as first cover for burn patients. Adv Wound Care (New Rochelle) 8(11): 555-564.

26. Foglia RP, Di Preta J, Statter MB, Donahoe PK (1986) Fetal allograft survival in immunocompetent recipients is age dependent and organ specific. Ann Surg 204(4): 402-410.

27. Gabbianelli M, Sargiacomo M, Pelosi E, Testa U, Isacchi G, Peschle C (1990) "Pure" human hematopoietic progenitors: Permissive action of basic fibroblast growth factor. Science 249(4976): 1561-1564.
28. Le Blanc K, Tammik C, Rosendahl K, Zetterberg E, Ringdén O (2003) HLA expression and immunologic properties of differentiated and undifferentiated mesenchymal stem cells. Exp Hematol 31(10): 890896.

29. Devine SM, Bartholomew AM, Mahmud N, Nelson M, Patil S, Hardy W, et al. (2001) Mesenchymal stem cells are capable of homing to the bone marrow of non-human primates following systemic infusion. Exp Hematol 29(2): 244-255.

30. Bartholomew A, Sturgeon C, Siatskas M, Ferrer K, McIntosh K, et al. (2002) Mesenchymal stem cells suppress lymphocyte proliferation in vitro and prolong skin graft survival in vivo. Exp Hematol 30(1): 42-48.

31. Streit M, Braathen LR (2000) Apligraf--A living human skin equivalent for the treatment of chronic wounds. Int J Artif Organs 23(12): 831-833.

32. Tsujisaki M, Igarashi M, Sakaguchi K, Eisinger M, Herlyn M, et al. (1987) Immunochemical and functional analysis of HLA class II antigens induced by recombinant immune interferon on normal epidermal melanocytes. J Immunol 138(4): 1310-1316.

33. Piccinni MP (2010) T cell tolerance towards the fetal allograft. J Reprod Immunol 85(1):71-5.

34. Deschaseaux F, Delgado D, Pistoia V, Giuliani M, Morandi F, et al. (2011) HLA-G in organ transplantation: Towards clinical applications. Cell Mol Life Sci 68(3): 397-404.

35. Zuliani T, Saiagh S, Knol AC, Esbelin J, Dréno B (2013) Fetal fibroblasts and keratinocytes with immunosuppressive properties for allogeneic cell-based wound therapy. PLoS One 8(7): e70408.

36. Rouas-Freiss N, Marchal RE, Kirszenbaum M, Dausset J, Carosella ED (1997) The alpha1 domain of HLA-G1 and HLA-G2 inhibits cytotoxicity induced by natural killer cells: Is HLA-G the public ligand for natural killer cell inhibitory receptors? Proc Natl Acad Sci USA 94(10): 52495254.

37. Ober C (1998) HLA and pregnancy: The paradox of the fetal allograft. Am J Hum Genet 62(1): 1-5.

38. Carosella ED, Moreau P, Lemaoult J, Rouas-Freiss N (2008) HLA-G: From biology to clinical benefits. Trends Immunol 29(3): 125-132.

39. Santos MA, O’Donoghue K, Wyatt-Ashmead J, Fisk NM (2008) Fetal cells in the maternal appendix: A marker of inflammation or fetal tissue repair? Hum Reprod 23(10): 2319-2325. 